\title{
On the diagonalization of the measure matrix in the eigenfunction expansion for ordinary differential equations of the second order
}

\author{
by \\ Yoshimi SAItō
}

Let us start with the outline of the theory of the eigenfunction expansion for the second order differential operator due to $\mathrm{H}$. Weyl, M. H. Stone, E. C. Titchmarsh and K. Kodaira. Consider a differential expression with suitable boundary conditions

$$
L=-\frac{d}{d x}\left(p(x) \frac{d}{d x}\right)+q(x)
$$

defined in an open interval $(a, b)$ and take any linearly independent system of solutions $\left(s_{1}(x, \lambda), s_{2}(x, \lambda)\right.$ of $L(u)=\lambda u$ such that each $s_{i}(x, \lambda)$ is extendable to an entire function of $\lambda$ for every $x$. Using $s_{1}(x, \lambda)$ and $s_{2}(x, \lambda)$, we can construct a $(2,2)$-matrix $\boldsymbol{P}(\lambda)=\left(\rho_{i j}(\lambda)\right)$ depending on $\lambda$ with is called the measure matrix. Writing

$$
L_{2}(d \boldsymbol{P}(\lambda))=\left\{\phi(\lambda)=\left(\phi_{1}(\lambda), \phi_{2}(\lambda) /\|\phi\|<\infty\right\}\right.
$$

with

$$
\|\phi\|^{2}=\sum_{i, j=1,2} \int_{-\infty}^{\infty} \phi_{i}(\lambda) \overline{\phi_{j}(\lambda)} d \rho_{i j}(\lambda),
$$

we obtain an isonorphism:

$$
\left(L_{2}(a, b) ; d x\right)=L_{2}(d \boldsymbol{P}(\lambda))
$$

where the corresponding $f(x)$ and $\left(\phi_{1}(\lambda), \phi_{2}(\lambda)\right)$ and are obtained from each other by

$$
f(x)=\sum_{i, j=1,2} \int_{=\infty}^{\infty} \phi_{i}(\lambda) s_{j}(x, \lambda) d \rho_{i j}(\lambda)
$$

Received Octover 12 


$$
\phi_{i}(\lambda)=\int_{a}^{b} f(x) s_{i}(x, \lambda) d x \quad i=1,2 .
$$

It is easy to see that any linearly independent system of solutions $\left(t_{1}(x, \lambda), t_{2}(x, \lambda)\right)$, each $t_{i}(x, \lambda)$ being extendable to an entire function of $\lambda$, is obtained by the transformation

$$
t_{i}(x, \lambda)=\sum_{j=1}^{2} a_{i j}(\lambda) s_{j}(x, \lambda) \quad i=1,2
$$

and vice versa. where each $\left.a_{i}, \lambda\right)$ is extendable to an entire function of $\lambda$ and $\operatorname{det}\left(a_{i j}\right)$ never vanishes in the complex $\lambda$-plane. $\phi_{i}(\lambda)$ and $d \rho_{i j}(\lambda)$ are transformed by each transformation as follows:

$$
\phi_{i}(\lambda) \rightarrow \psi_{i}(\lambda)=\sum_{l=1}^{2} a_{i l}(\lambda) \phi_{l}(\lambda)
$$

and

$$
d \rho_{i j}(\lambda) \rightarrow d \sigma_{i j}(\lambda)=\sum_{k, l=1,2} a_{i k}(\lambda) a_{j l}^{-1}(\lambda) d \rho_{k l}(\lambda),
$$

where $\left(a_{i j}^{-1}(\lambda)\right)$ is the inverse matrix of $\left(a_{i j}(\lambda)\right)$.

Even if we take instead of $a_{i j}(\lambda), i, j=1,2$ measurable functions $b_{i j}(\lambda), i, j=1,2$ with $\operatorname{det}\left(b_{i j}(\lambda)\right) \neq 0$, the $t_{i}(x, \lambda), i=1,2$ obtained above will form a linearly independent system of solutions of $L(u)=\lambda u$ and we have the same isomorphism as above, though each $t_{i}(x, \lambda)$ is not necessarily extendable to an entire function of $\lambda$ any more.

Let us call for the former transformation by $\left(a_{i j}(\lambda)\right)$ analytic and the latter one by $\left(b_{i j}(\lambda)\right)$ measurable. As is well-known, $d \sigma_{i j}(\lambda)$ turns out to be diagonal by a suitable measurable transformation, see Dunford and Schwartz [3] XII, 5, while such a diagonalization is not always possible by analytic transformations.

The aim of the present paper is to discuss the diagonalization of the measure matrix by analytic transformations that will be called the analytic diagonalization. If the measure matrix for $L$ is analytically diagonalizable we simply say that $L$ is analytically diagonalizable.

In Section 1 we shall give a necessary and sufficient condition for $L$ to be analytically diagonalizable. In Section 2 we shall use this to construct an example of $L$ which is not analytically diagona- 
lizable and to see that the case of symmetric potentials is a typical example which is analytically diagonalizable. In section 3 we shall give some examples which can be obtained by purturbating symmetric potentiais and are still analytically diagonalizable.

We shall follow K. Kodaira [7] as to the notations.

I want to thank professor $\mathrm{K}$. Ito for his interest given to this work.

\section{\$1. A condition for the possibility of the analytic diagonalization of $\mathbb{L}$.}

Given a differential expression:

$$
L=-\frac{d}{d x}\left(p(x) \frac{d}{d x}\right)+q(x) \quad(a<x<b,-\infty \leqq a<b \leqq \infty)
$$

defined in a (finite or infinite) interval $(a, b)$, where $p(x), q(x)$ are real-valued functions defined in $(a, b), p(x)$ has continuous first derivative, $q(x)$ is continuous and $p(x)>0$ for $a<x<b$, let us consider the differential equation

$$
L(u)=l u,
$$

where $l$ means a complex parameter.

By a system of fundamental solutions we shall mean a system of two solutions $s_{1}(x, l), s_{2}(x, l)$ of the equation $L(u)=l u$ having the following four properties:

$$
\begin{cases}\text { i) } & {\left[s_{2}, s_{1}\right]=l} \\ \text { ii) } & s_{k}(x, \bar{l})=\overline{s_{k}(x, l)} k=1,2 \text { in the whole } l \text {-plane, } \\ \text { iii) } & \text { as a function of }(x, l), s_{k}(x, l) \text { and }(d / d x) s_{k}(x, l) \\ & k=1,2 \text { are continuous. } \\ \text { iv) } & \text { as a function of } l, s_{k}(x, l) \text { and }(d / d x) s_{k}(x, l) k=1,2 \\ & \text { are entire for every } x,\end{cases}
$$

where $\left[s_{2}, s_{1}\right]$ is a bracket

$$
\begin{aligned}
{\left[s_{2}, s_{1}\right\rfloor=} & p(x)\left(s_{2}(x, l) s_{1}^{\prime}(x, l)-s_{1}(x, l) s_{2}^{\prime}(x, l)\right) \\
& \left.\left(s_{k}^{\prime}=d / d x\right) s_{k}, k=1,2\right)
\end{aligned}
$$


and the bar means the conjugate complex number.

For a system of fundamental solutions there exists a pair of functions of $l\left(f_{a}(l), f_{b}(l)\right)$ having the following properties:

$$
\left\{\begin{array}{l}
\text { i) } f_{a} \overline{(l)}=\overline{f_{a}(l)}, \overline{f_{b}(l)}=f_{b}(l) \text { in the whole } l \text {-plane, } \\
\text { ii) } f_{a}(l) \neq f_{b}(l) \text { for } \operatorname{Im} l \neq 0, \\
\text { iii) } f_{a}(l) \text { and } f_{b}(l) \text { are meromorphic in } \operatorname{Im} l \neq 0 .
\end{array}\right.
$$

The functions $f_{a}(l)$ and $f_{b}(l)$ will be called characteristic functions.

With suitable boundary conditions at $a$ and $b, L$ is a self-adjoint differential operator in $L_{2}((a, b) ; d x)$. Then, choosing a system of characteristic functions $\left(f_{a}(l), f_{b}(l)\right)$ and choosing a pair of boundary conditions at $a$ and $b$ are equivalent to each other.

Define the characteristic matrix $M(l)$ by

$$
\boldsymbol{M}(l)=\left(\begin{array}{ll}
M_{11}(l) & M_{12}(l) \\
M_{21}(l) & M_{22}(l)
\end{array}\right)=\frac{1}{f_{a}(l)-f_{b}(l)}\left(\begin{array}{lc}
f_{a}(l) f_{b}(l) & f_{a}(l)+f_{b}(l) \\
f_{a}(l)+f_{b}(l) & 1
\end{array}\right)
$$

and the matrix $\boldsymbol{P}(\lambda)=\left(\rho_{i j}(\lambda)\right)$ by

$$
\boldsymbol{P}(\lambda)=\left(\begin{array}{ll}
\rho_{11}(\lambda) & \rho_{12}(\lambda) \\
\rho_{21}(\lambda) & \rho_{22}(\lambda)
\end{array}\right)=\lim _{\delta \rightarrow+0} \lim _{\varepsilon \rightarrow+0} \frac{1}{\pi} \int_{\delta}^{\lambda+\delta} \operatorname{Im}[\boldsymbol{M}(\mu+i \varepsilon)] d \mu
$$

The measure matrix $d \boldsymbol{P}(\lambda)$ is positive semi-definite and symmetric.

Let $H$ denote the Hilbert space $L_{2}((a, b) ; d x)$ where the norm of $u$ will be denoted by $\|u\|$. Next, we consider $\lambda$-measurable vector functions $\phi(\lambda)=\left(\phi_{1}(\lambda), \phi_{2}(\lambda)\right)$ and put

$$
\|\phi\|_{*}^{2}=\int_{-\infty}^{\infty} \sum_{i, j=1,2} \phi_{i}(\lambda) \overline{\phi_{j}(\lambda)} d \rho_{i j}(\lambda) .
$$

Then $H^{*}=\left\{\phi /\|\phi\|_{*}<\infty\right\}$ constitutes a Hilbert space, and the transformation

$$
u(x) \rightarrow \phi_{i}(\lambda)=\int_{a}^{b} \operatorname{si}(x, \lambda) u(x) d x \quad i=1,2
$$

proves to be a unitary transformation from $H$ onto $H^{*}$, whose inverse is given by

$$
\phi_{i}(\lambda) \rightarrow u(x)=\int_{-\infty}^{\infty} \sum_{j k=1,2} s j(x, \lambda) \phi_{k}(\lambda) d \rho_{j k}(\lambda)
$$


Now we introduce a new definition:

Definition. A differential expression $L$ in (1.1) will be analytically diagonalizable if there exists a system of fundamental solutions $\left(s_{1}(x, l), s_{2}(x, l)\right)$ and a system of characteristic functions $\left(f_{a}(l)\right.$, $\left.f_{b}(l)\right)$ of $\left(s_{1}(x, l), s_{2}(x, l)\right)$ such that the measure matrix defined by (1.4) is a diagonal matrix for any real number $\lambda$.

For the sake of simplicity we shall say that $L$ is diagonalizable when $L$ is analytically diagonalizable.

For any $\nu>0$ the characteristic matrix $M(l)$ can be represented as follows:

$$
M(l)=\int_{-\nu}^{\nu} \frac{d \boldsymbol{P}(\lambda)}{\lambda-l}+\mathbb{R}^{(\nu)}(l),
$$

where $\boldsymbol{R}^{(v)}(l)$ is a (2.2)-matrix function whose components are regular on the complex plane with the slits $l \leqq-\nu$ and $l \geqq \nu$.) Let us assume that $\boldsymbol{P}(\lambda)$ is a diagonal matrix for any $\lambda$. Then we have

$$
\left\{\begin{array}{l}
M_{12}(l)=R_{12}^{(\nu)}(l) \\
M_{21}(l)=R_{21}^{(\nu)}(l)
\end{array}\right.
$$

As the real number $\nu$ is arbitrary, $M_{12}(l), M_{21}(l)$ are regular functions in the whole $l$-plane. Therefore it follows from (1.3) that

$$
\frac{f_{a}(l)+f_{b}(l)}{f_{a}(l)-f_{b}(l)}=\frac{1+f_{b}(l) / f_{a}(l)}{1-f_{b}(l) / f_{a}(l)}
$$

is an entire function i. e. $f_{a}(l) / f_{a}(l)$ is a meromorphic function in the whole $l$-plane and $f_{b}(l) / f_{a}(l) \neq 1$ for any complex $l$.

Conversely, if $f_{b}(l) / f_{a}(l)$ is meromorphic in the whole $l$-plane and $f_{b}(l) / f_{a}(l) \neq 1$, then $M_{12}(l)$ and $M_{21}(l)$ are regular. Hence we have

$$
\rho_{12}(\lambda)=\rho_{21}(x)=0 \quad(-\infty<\lambda<\infty) .
$$

Thus we obtain

Lemma 1. 1. In order that $L$ is diagonalizable it is necessary and sufficient that exists a system of characteristic functions $\left(f_{a}\right.$,

1) [7]. p. $932 .(2.15)$. 
$f_{b}$ ) of $\left(s_{1}, s_{2}\right)$ such that $f_{b}(l) / f_{a}(l)$ is meromorphic in the whole $l$-plane and $f_{b}(l) / f_{a}(l) \neq 1$ for any complex $l$.

Let us consider two systems of fundamental solutions $\left(s_{1}^{0}, s_{2}^{0}\right)$ and $\left(s_{1}, s_{2}\right)$. Then there exists a $(2,2)$-matrix function $\mathbb{A}(l)=\left(a_{i j}(l)\right)$ such that

$$
\begin{cases}\text { i) } & \boldsymbol{A}(\bar{l})=\overline{\boldsymbol{A}(l)} \\ \text { ii) } & a_{i j}(l) i, j=1,2 \text { are entire function, } \\ \text { iii) } \operatorname{det} \boldsymbol{A}(l)=1 \text { in the } l \text {-plane }\end{cases}
$$

and $s_{1}, s_{2}$ are related to $s_{1}^{0}, s_{2}^{0}$ by a unimodular transformation $A(l)$

$$
\left(\begin{array}{l}
s_{1}^{0}(x, l) \\
s_{2}^{0}(x, l)
\end{array}\right)=\mathbb{A}^{t}(l)\left(\begin{array}{l}
s_{1}(x, l) \\
s_{2}(x, l)
\end{array}\right),
$$

where $\boldsymbol{A}^{t}(l)$ is the transposed matrix of $\boldsymbol{A}(l)$. Denote by $\left(f_{a}, f_{b}\right)$ (or $\left(f_{a}^{0}, f_{b}^{0}\right)$ ) the system of characteristic functions of $\left(s_{1}^{0}, s_{2}^{0}\right)$ ) (or $\left.\left(s_{a}^{0}, s_{0}^{b}\right)\right)$. Then $\left(f_{a}, f_{b}\right)$ is related to $\left(f_{a}^{0}, f_{b}^{0}\right)$ by a linear transformation $\boldsymbol{A}(l)$ :

$$
\begin{aligned}
& f_{a}(l)=\boldsymbol{A}(l) f_{a}^{0}(l) \\
& f_{b}(l)=\boldsymbol{A}(l) f_{b}^{0}(l),
\end{aligned}
$$

where we put

$$
\boldsymbol{A}(l) f(l)=\frac{a_{11}(l) f(l)+a_{12}(1)}{a_{21}(l) f(1)+a_{22}(l)} .
$$

Using these facts we can restate the lemma 1.1 as follows:

Lemma 1.2. In order that $L$ is diagonalizable it is necessary and sufficient that for any arbitrary system of characteristic functions $\left(f_{a}, f_{b}\right)$ there exists $a(2,2)$-matrix function $A_{0}(l)=\left(A_{i j}(l)\right)$ such that $f_{b}(l)=\boldsymbol{A}_{0}(l) f_{a}(l)$. Here $\boldsymbol{A}_{0}(l)$ satisfies the following condittons:

$$
\left\{\begin{aligned}
\text { i ) } & A_{i j}(l) i, j=1,2 \text { are meromorphic in the whole l-plane, } \\
\text { ii }) & \boldsymbol{A}_{0}(\bar{l})=\overline{\boldsymbol{A}_{0}(l)} \\
\text { iii }) & \text { if we put } \operatorname{det} \boldsymbol{A}_{0}(l)=f(l) \text {, then } f(l) \neq 1 \text { and } A_{0}(l) / \\
& (1-f(l)) \text { is regular in the whole l-plane, } \\
\text { iv) } & A_{11}(l)+A_{2 z}(l)=f(l)+1 \text { for any complex } l .
\end{aligned}\right.
$$


If for an arbitrary $\left(f_{a}(l), f_{b}(l)\right)$ there exists such $A_{0}(l)$, then we can choose a suitable system of characteristic functions $\left(f_{a}^{0}(l)\right.$, $f_{b}^{0}(l)$ such that

$$
f_{b}^{0}(l) / f_{a}^{0}(l)=f(l) .
$$

As the proof is easy, we omit it.

\section{§2. The Potential which is not diagonalizable and the symmetric potential.}

In this section and the next section we shall assume with no loss of generality that $p(x) \equiv 1$ and $(a, b)=(-\infty, \infty)$, and call $q(x)$ in (1.1) the potential of $L$. Moreover we assume that $L$ is of the limit point type both at $-\infty$ and at $+\infty$, for $L$ is always diagonalizable if $L$ is of the limit circle type either at $-\infty$ or at $\infty$.

We divide the differential operator $L$ into the following two differential operators in $[0, \infty]$ :

$$
\begin{cases}L_{1}=-\frac{d^{2}}{d x^{2}}+q_{1}(x), & q_{1}(x)=q(x) \\ L_{2}=-\frac{d^{2}}{d x^{2}}+q_{2}(x), & q_{2}(x)=q(-x) .\end{cases}
$$

$L_{1}$ and $L_{2}$ are regular at $x=0$ and their spectra are simple.

Denote by $\left(s_{1}^{0}, s_{2}^{0}\right)$ the system of fundamental solutions of $L$ satisfying the following conditions:

$$
\left\{\begin{array}{l}
s_{1}(0, l)=s_{2}^{\prime}(0, l)=1 \\
s_{1}^{\prime}(0, l)=s_{2}(0, l)=0
\end{array} \quad \text { for all } l .\right.
$$

Any system of fundamental solutions satisfying (2.2) will be called the natural system of fundamental solutions. Denote by $\left(f_{a}^{0}, f_{b}^{0}\right)$ the system of characteristic functions of $\left(s_{1}^{0}, s_{2}^{0}\right)$. Then we define the system of fundamental solutions $\left(s_{1}^{0}, s_{2}^{0}\right)$ of $L_{1}$ by

$$
\left\{\begin{array}{l}
s_{1}^{(1)}(x, l)=s_{1}^{0}(x, l) \\
s_{2}^{(1)}(x, l)=s_{2}^{0}(x, l)
\end{array} \quad(0 \leqq x<\infty)\right.
$$


and the system of fundamental solutions $\left(s_{1}^{(2)}, s_{2}^{(2)}\right)$ of $L_{2}$ by

$$
\left\{\begin{array}{l}
s_{1}^{(2)}(x, l)=s_{1}^{0}(-x, 1) \\
s_{2}^{(2)}(x, l)=-s_{2}^{0}(-x, l) .
\end{array} \quad(0 \leqq x<\infty)\right.
$$

Denoting by $f_{1}(l)$ and $f_{2}(l)$ the characteristic functions at $\infty$ of $\left(s_{1}^{(1)}, s_{2}^{(1)}\right)$ and $\left(s_{1}^{(2)}, s_{2}^{(1)}\right)$ respectively, we clearly obtain

$$
\left\{\begin{array}{l}
f_{1}(l)=f_{\infty}^{0}(l) \\
f_{2}(l)=-f_{-\infty}^{0}(l) .
\end{array}\right.
$$

Let us assume that $L$ is diagonalizable. Then there exists a system of characteristic functions $\left(f_{-\infty}^{*}(l), f_{\infty}^{*}(l)\right)$ such that $f_{\infty}^{*} / f_{-\infty}^{*}$ is meromorphic. Therefore denoting by $\mathfrak{U}_{\infty}^{*}$ (or $\mathfrak{U}_{-\infty}^{*}$ ) the set of real numbers $\lambda$ such that $f_{\infty}^{*}\left(\right.$ or $f_{-\infty}^{*}$ ) is not meromorphic at $\lambda$, we obtain

$$
\mathfrak{A}_{\infty}^{*}=\mathfrak{U}_{-\infty}^{*} \text {. }
$$

As $f_{\infty}^{*}$ (or $f_{-\infty}^{*}$ ) is related to $f_{\infty}^{0}$ (or $f_{-\infty}^{0}$ ) by a linear transformation with the components regular in $l$, it follows from (2.4) that

$$
\mathfrak{P}_{\infty}^{0}=\mathfrak{A}_{-\infty}^{0}
$$

where $\mathfrak{A}_{\infty}^{0}$ (or $\mathfrak{A}_{-\infty}^{0}$ ) is the set of real mumbers $\lambda$ such that $f_{\infty}^{0}$ (or $f_{-\infty}^{0}$ ) is not meromorphic at $\lambda$. From (2.3) we also obtain

$$
\mathfrak{U}_{1}=\mathfrak{U}_{2}
$$

where $\mathfrak{U}_{1}$ (or $\mathfrak{U}_{2}$ ) is the set of real numbers $\lambda$ such that $f_{1}$ (or $f_{2}$ ) is not meromorphic at $\lambda$. It well-known that the essential spectrum of $L_{1}$ (or $L_{2}$ ) is independent of the special choice of its boundary conditions. ${ }^{2}$ Therefore it follows from the spectral formula (1.4) that $\mathfrak{A}_{1}$ (or $\mathfrak{U}_{2}$ ) is the essential spectrum of $L_{1}$ (or $L_{2}$ ). Using (2.6) we obtain

Theorem 2.1. If $L$ is of limit point type at both $-\infty$ and $\infty$ and $L$ is diagonalizable, then the essential spectrum of $L_{1}$ and $L_{2}$ in (2.1) coincide with each other.

By virtue of the theorem 2.1 we can construct a differential

2) [3]. p. 1394. 
expression $L$ which is not diagonalizable. Take for example a function $\varphi(x)$ continuous on $[0, \infty)$ and that for a real number $k \neq 0|\varphi(x)-k|$ is summable in $(0, \infty)$ and $\varphi(0)=0$. If we put

$$
q(x)= \begin{cases}\varphi(x) & (x \geqq 0) \\ 0 & (x<0),\end{cases}
$$

we obtain ${ }^{3)}$

$$
\begin{aligned}
& \mathfrak{A}_{1}=[k, \infty) \\
& \mathfrak{A}_{2}=[0, \infty) .
\end{aligned}
$$

Hence $\mathfrak{A}_{1} \neq \mathfrak{A}_{2}$, so that $L$ is not diagonalizable.

Next, let us consider the case that the potential $q(x)$ is symmetric i. e. $q(x)=q(-x)$. In this case we have

$$
f_{\infty}^{0}(l)+f_{-\infty}^{0}(l)=0,
$$

which shows that $L$ is diagonalizable by the natural system of fundamental solutions.

Conversely, let us assume that $L$ is diagonalizable by a natural system of fundamental solution $\left(s_{1}^{0}, s_{2}^{0}\right)$. Taking $\left(f_{-\infty}^{0}, f_{\infty}^{0}\right)$ as above and denoting with $d \boldsymbol{P}^{0}(\lambda)=\left(d \rho_{i j}^{0}(\lambda)\right)$ the measure matrix defined by $\left(f_{-\infty}^{0}, f_{\infty}^{0}\right)$, we obtain

$$
0=\int_{-\infty}^{\infty} \frac{d \rho_{12}^{0}(\lambda)}{|\lambda-l|^{2}}=\int_{-\infty}^{\infty} \overline{r_{1}(x, l)} r_{2}(x, 1) d x^{4)}
$$

where

$$
\left\{\begin{aligned}
r_{1}(x, l) & = \begin{cases}w_{\infty}(x, l)\left[f_{-\infty}^{0}(l)-f_{\infty}^{0}(l)\right]^{-1} & (x \geqq 0) \\
w_{-\infty}(x, l)\left[f_{-\infty}^{0}(l)-f_{\infty}^{0}(l)\right]^{-1} & (x \leqq 0),\end{cases} \\
r_{2}(x, l) & = \begin{cases}-f_{\infty}^{0}(l) w_{\infty}(x, l)\left[f_{-\infty}^{0}(l)-f_{\infty}^{0}(l)\right]^{-1} & (x \geqq 0) \\
-f_{-\infty}^{0}\left(w_{-\infty}(x, l)\left[f_{-\infty}^{0}(l)-f_{\infty}^{0}(l)\right]^{-1}\right. & (x \leqq 0),\end{cases} \\
w_{\infty}(x, l) & =s_{2}^{0}(x, l)+f_{\infty}^{0}(l) s_{1}^{0}(x, l), \\
w_{-\infty}(x, l) & =s_{2}^{0}(x, l)+f_{-\infty}^{0}(l) s_{1}^{0}(x, l) .
\end{aligned}\right.
$$

From (2.7) we obtain

3) [9]. p. 115-119.

4) $[7]$. p. $931,(2.12)$. 


$$
f_{\infty}^{0}(l) / f_{-\infty}^{0}(l)=-\frac{\int_{0}^{\infty}\left|w_{\infty}(x, l)\right|^{2} d x}{\int_{-\infty}^{0}\left|w_{-\infty}(x, l)\right|^{2} d x}<0
$$

and $f_{\infty}^{0}(l) / f_{-\infty}^{0}(l)$ is meromorphic, so that

$$
f_{\infty}^{0}(l) / f_{-\infty}^{0}(l)=\text { const. }
$$

On the other hand we have

$$
\lim _{t \rightarrow \infty} \frac{f_{1}(i t)}{f_{2}(i t)}=1
$$

in general, where $t$ is a real number.5) Therefore we obtain

$$
f_{1}(l) \equiv f_{2}(l) \quad \text { or } \quad f_{\infty}^{0}(l) / f_{\infty-}^{0}(l)=-1 .
$$

In addition to this fact, the characteristic function $f_{1}(l)$ (or $f_{2}(l)$ ) uniquely determines the potential $q_{1}(x)$ (or $q_{2}(x)$ ). ${ }^{6}$ ) Hence in this case we have

$$
q_{1}(x)=q_{2}(x) \quad \text { i. e. } \quad q(x)=q(-x) .
$$

Theorem 2.2. If $L$ is diagonalizable by the natural system of fundamental solutions, then $q(x)$ is symmetric i.e. $q(x)=q(-x)$.

\section{§3. Examples of $\mathbb{Z}$ which is diagonalizable.}

From the result of Section 2 we know the following: If the potential $q(x)$ is not symmetric at infinity, for example the limits of $q(x)$ exist and

$$
\lim _{\tau \rightarrow \infty} q(x) \neq \lim _{x \rightarrow-\infty} q(x),
$$

$L$ is not diagonalizable. The symmetric potential $q(x)=q(-x)$ is a typical potential which makes $L$ diagonalizable.

These fact will suggest the following question: In case the potential $q(x)$ of $L$ is obtained by suitable perturbation of a sym-

5) [1]. p. $285,[6]$. p. $338,[9]$. p. 119.

6) [1]. Lemma 1. 
metric potential, is $L$ diagonalizable? we cannot give a complete answer to this question, but we shall discuss some examples which might suggest the solution more or less.

Let us consider two potentials $q(x)$ and $q_{0}(x)$ in $(-\infty,+\infty)$. We put

$$
\left\{\begin{array}{l}
L=-\frac{d^{2}}{d x^{2}}+q(x) \\
L_{0}=-\frac{d}{d x^{2}}+q_{0}(x) .
\end{array}\right.
$$

Let $\left(s_{1}^{0}, s_{2}^{0}\right)$ (or $\left(t_{1}^{0}, t_{2}^{0}\right)$ ) denote the natural system of fundamental solutions of $L$ (or $\left.L_{0}\right)$ and $\left(f_{-\infty}^{0}, f_{\infty}^{0}\right)$ (or $\left(g_{-\infty}^{0}, g_{\infty}^{0}\right)$ ) the system of characteristic functions of $\left(s_{1}^{0}, s_{2}^{0}\right)$ (or $\left(t_{1}^{0}, t_{2}^{0}\right)$ ). Now we impose the following assumption: There exist two positive constants $k$ and $\varepsilon$ such that

$$
\left|q(x)-q_{0}(x)\right| \exp \left|k\left(x+\int_{0}^{x}|q(y)| d y\right)^{1+\varepsilon}\right|
$$

and

$$
\left.\left|q(-x)-q_{0}(-x)\right| \exp \mid k\left(x+\int_{0}^{x}|q(-y)| d y\right)^{1+\varepsilon}\right]
$$

are summable in $(0, \infty)$.

Under this assumption, we easily see by the general theory of ordinary differential equations that there exist two $(2,2)$-matrix functions $\mathbb{A}_{-\infty}(l)$ and $\mathbb{A}_{\infty}(l)$ satisfying (1.5) such that

$$
f_{ \pm \infty}^{0}(l)=\boldsymbol{A}_{ \pm \infty}(l) g_{ \pm \infty}^{0}(l)^{7)}
$$

Therefore putting $\boldsymbol{A}_{0}(l)=\boldsymbol{A}_{-\infty}^{t}(l) A_{\infty}(l), \quad\left(g_{-\infty}^{0}, \boldsymbol{A}_{0} g_{\infty}^{0}\right)$ is a system of characteristic functions of $L$. Let us assume further that $q_{0}(x)=q_{0}(-x)$. Putting

$$
-g_{-\infty}^{0}=g_{\infty}^{0} \equiv g
$$

we see that $\left(-g, \boldsymbol{A}_{0} g\right)$ is a system of characteristic functions of $L$. In order to show that $L$ is diagonalizable, it is sufficient to show that there exist a $(2,2)$-matrix function $\boldsymbol{A}(l)$ satisfying (1.5) such that

7) [2]. §2, §3. [9]. p. 115-119. 


$$
\mathbb{A}(l)(-g(l)) / \mathbb{A}(l) \mathbb{A}_{0}(l) g(l)
$$

is meromorphic and never takes the value 1. Putting

$$
\begin{aligned}
& \mathbb{A}_{0}(l)=\left(\begin{array}{ll}
A(l) & C(l) \\
B(l) & D(l)
\end{array}\right), \quad \mathbb{A}(l)=\left(\begin{array}{ll}
\alpha(l) & \gamma(l) \\
\beta(l) & \delta(l)
\end{array}\right), \\
& \mathbb{A}^{*}(l)=\mathbb{A}(l) \mathbb{A}_{0}(l)=\left(\begin{array}{ll}
\alpha^{*}(l) & \gamma^{*}(l) \\
\beta^{*}(l) & \delta^{*}(l)
\end{array}\right), \\
& \mathbb{A}(-g)=f_{-\infty}^{*}, \quad \mathbb{A}^{*} g=f_{\infty}^{*},
\end{aligned}
$$

we have

$$
\left\{\begin{array}{l}
f_{\infty}^{*}=\frac{(\alpha A+\gamma B) g+(\alpha C+\gamma D)}{(\beta A+\delta B) g+(\beta C+\delta D)}=\frac{\alpha^{*} g+\gamma^{*}}{\beta^{*} g+\delta^{*}}, \\
f_{-\infty}^{*}=\frac{\alpha(-g)+\gamma}{\beta(-g)+\delta} .
\end{array}\right.
$$

Theorem 3.1. (1) If there exist two positive numbers $k$ and $\varepsilon$ such that $|q(x)| \exp \left\{k|x|^{1+\varepsilon}\right\}$ is summable in $(-\infty, \infty), L$ is diagonalizable.

(2) If there exist two positive mumbers $k$ and $\varepsilon$ such that $\left|q(x)+x^{2}\right| \exp \left\{k|x|^{3+\varepsilon}\right\}$ is summable in $(-\infty, \infty), L$ is diagonalizable.

Corollary. If $q(x)$ has a compact support, $L$ is diagonalizable.

The case (1) occurs when $q_{0}(x)=0$ and the case (2) occurs when $q_{0}(x)=-x^{2}$. Clearly $L$ has double spectra in both cases. First we prove the case (1). If $q_{0}(x)=0, g(l)=-i \sqrt{ } \bar{l}$. In order that $f_{\infty}^{*} / f_{-\infty}^{*}$ is meromorphic, it is necessary that

$$
\operatorname{Im}\left(f_{\infty}^{*} / f_{-\infty}^{*}\right)=0,
$$

for any real $\lambda$ that belongs to the essential sepectrum of $L$. Let

$$
\left(\begin{array}{ll}
a(l) & c(l) \\
b(l) & d(l)
\end{array}\right)
$$

be a $(2,2)$-matrix function satisfying (1.5). Then we have

$$
\frac{a( \pm i \sqrt{l})+c}{b( \pm i \sqrt{l})+d}=\frac{c d+a b l \pm i \sqrt{l}}{d^{2}+b^{2} l} .
$$

Hence it follows from (3.1) that for $\lambda$ in $[0, \infty)$ 


$$
\sqrt{\lambda}\left\{\gamma^{*} \delta^{*}+\alpha^{*} \beta^{*} \lambda\right\}-(-\sqrt{\lambda})\{\gamma \delta+\alpha \beta \lambda\}=0,
$$

i. e. for any complex $l$

$$
\gamma^{*}(l) \delta^{*}(l)+\alpha^{*}(l) \beta^{*}(l) l+\gamma(l) \delta(l)+\alpha(l) \beta(l) l=0 .
$$

Conversely if (3.2) is satisfied, we have

$$
f_{\infty}^{*} / f_{-\infty}^{*}=-\frac{\delta^{2}+l \beta^{2}}{\delta^{* 2}+l \beta^{* 2}},
$$

i.e. $f_{\infty}^{*} / f_{-\infty}^{*}$ is meromorphic. Therefore, determining the $\boldsymbol{A}(l)$ so that it satisfies (3.2) and $\alpha \delta-\beta \delta=1$, we have

$$
\left\{\begin{array}{l}
\alpha=\frac{\delta K+\beta L}{\delta^{2} K+2 \beta \delta L+\beta^{2} M} \\
\gamma=\frac{\delta L+\beta M}{\delta^{2} K+2 \beta \delta L+\beta^{2} M},
\end{array}\right.
$$

where

$$
f_{-\infty}^{*} / f_{\infty}^{*}=1-\frac{\delta^{2} K+2 \beta \delta L+\beta^{2} M}{\delta^{2}+l \beta^{2}}
$$

$$
\left\{\begin{array}{l}
K(l)=1+D(l)^{2}+B(l)^{2} l \\
L(l)=C(l) D(l)+A(l) B(l) l \\
M(l)=C(l)^{2}+\left(A(l)^{2}+1\right) l,
\end{array}\right.
$$

which depend only on the potential $q(x)$.

Now let $\beta(l)$ and $\delta(l)$ be two arbitrary entire functions satisfying $\beta \overline{(l)}=\overline{\beta(l)}$ and $\delta(\bar{l})=\overline{\delta(l)}$. Then $\alpha(l)$ and $\gamma(l)$ determined by (3.3) are meromorphic functions satisfying $\alpha \overline{(l)}=\overline{\alpha(1)}$ and $\gamma \overline{(l)}=\overline{\gamma(l)}$ and

$$
f_{\infty}^{*}(l) / f_{-\infty}^{*}(l) \neq 1
$$

except for real $l$ such that $l \leqq 0$. But we must choose suitable entire functions $\beta(l)$ and $\delta(l)$ to satisfy (1.5) and (3.5) in the whole $l$-plane. Clearly the real number $\lambda \leqq 0$ such that $f_{\infty}^{*}(\lambda)=f_{-\infty}^{*}(\lambda)$ is an eigenvalue of $L$. In this case $\lambda=0$ is not an acummulating point of eigenvalues $\lambda_{n} n=1,2, \cdots$ which lie in $(-\infty, 0)$. Hence we can determine $\beta(l)$ and $\delta(l)$ as follows: Let $\left\{\lambda_{n}\right\}_{n=1,2} \cdots \quad\left(0>\lambda_{1}>\lambda_{2} \cdots\right)$ the eigenvalues in $(-\infty, 0)$ of $L$ and $\left\{\mu_{n}\right\}_{n=1,2} \cdots\left(0<\left|\mu_{1}\right| \leqq\left|\mu_{2}\right| \leqq \cdots\right)$ the root of the equation 


$$
K(l)^{2}\left\{M(l)^{2}+l K(l)^{2}+4 L\left(l^{2}\right)-2 K(l) M(l) l\right\}=0
$$

which do not belong to $\left\{\lambda_{n}\right\}$. Determine an entire function $\delta(l)$ such that

$$
\begin{cases}\text { i }) & \delta\left(\lambda_{n}\right)=-i \sqrt{\lambda_{n}} \text { and } \delta(l)+i \sqrt{\lambda_{n}} \text { has a simple root at } \\ & \lambda_{n}(n=1.2 \cdots), \\ \text { ii) } & \delta\left(\mu_{n}\right)^{2}+\mu_{n} \neq 0(n=1.2 \cdots) \\ \text { iii) } & \delta(\bar{l})=\overline{\delta(l)}\end{cases}
$$

and $\beta(l) \equiv 1$.

Then it is clear that

$$
A(l)=\left(\begin{array}{ll}
\alpha(l) & \gamma(l) \\
\beta(l) & \delta(l)
\end{array}\right)
$$

satisfies (1.5) and $\left(f_{-\infty}^{*}, f_{\infty}^{*}\right)$ satisfies (3.5) in the whole $l$-plane.

In the case (2) that $q_{0}(x)=-x^{2}$, the essential spectrum of $L_{0}$ is the whole real axis, so that the situation is much easier than the case (1); it is sufficient to put $\beta \equiv 0$ and $\delta \equiv 1$.

\section{BIBLIOGRAPHY}

[1] G. Borg, Uniqueness theorems in the spectral theory of $y^{\prime \prime}+(\lambda-q(\mathrm{x})) y=0,11$ Skand. Mat. Kongr., 276-287 (1952).

[2] L. Cesari, Asymptotic behavior and stability problems in ordinary differential equations, Spriger, Berlin (1962).

[3] N. Dunford-J. Schwartz, Liniear Operators, part $\mathbb{I}$, Interscience, New York (1963).

[4] H. Hahn, Uber die Integral des Herrn Hellinger und die Orthogonalinvarianten der quadratischen Formen von unendlich vielen Veränderlichen, Monatsh. Math.-Phys. 23, 161-224 (1912).

[5] E. Hellinger, Neue Beguendung der Theorie quadratischen Formen von unendlichvielen Veränderlichen, J. Reine Angew. Math. 136, 210-271 (1909).

[6] E. Hille, Green's transformation and singular boundary value problems, Math.. tome XLII-Fasc 4, 331-349 (1963).

[7] K. Kodaira, The eigenvalue problem for ordinary differential equations of the second order and Heisenberg's theory of S-matrix, Amer. J. Math. 71, 912-945 (1949).

[8] M. Stone, Linear transformations in Hilbert spaces and their applications to analysis, Amer. Math. Soc. Colloquim Pub. vol 15, New York (1932).

[9] E. C. Titchmarsh, Eigenfuction expansions associated with second-order differential equations, part I, Oxford Univ. Press, London (1961).

[10] H. Weyl, Ủber gewöhnliche Differentialgleichung mit Singularitäten und zugehörigen Entwicklungen willkürlicher Funktionen, Math. Ann. 68, 220-269 (1910). 